\title{
Can biologic treatment induce cutaneous focal mucinosis?
}

\author{
Aleksandra Lesiak, Marcin Włodarczyk, Aleksandra Sobolewska, Joanna Sieniawska, Michał Rogowski-Tylman, \\ Anna Sysa-Jedrzejowska, Irmina Olejniczak-Staruch, Joanna Narbutt
}

Department of Dermatology, Medical University of Lodz, Lodz, Poland

Head of the Department: Prof. Anna Sysa-Jedrzejowska MD, PhD

Postep Derm Alergol 2014; XXXI, 6: 413-416 DOI: $10.5114 /$ pdia.2014.40944

\begin{abstract}
Skin mucinosis is a rare skin disease which clinically manifests as firm papules and waxy nodules. We report a case of a 66-year-old female psoriatic patient who developed skin mucinosis during biological therapy. Because of a previous lack of response to the local and conventional systemic treatment of psoriasis, the patient received biological therapy (infliximab from June 2008 to May 2009 - initial clinical improvement and loss of treatment effectiveness in the $36^{\text {th }}$ week of the therapy; adalimumab from June 2009 to January 2010 - lack effectiveness; ustekinumab from March 2012 to the present). Throughout 2 months we observed a manifestation of the skin mucinosis as well-demarcated, yellow and brown, papulo-nodular lesions of 5-10 mm in diameter, localized on the back. Histopathological examination with alcian blue staining demonstrated mucin deposits in the dermis. On the basis of clinical and histopathological findings, the diagnosis of cutaneous focal mucinosis was established. We present the case because of the extremely rare occurrence of the disease. Scarce literature and data suggest that there is an association between focal mucinosis and thyroid dysfunction, as well as possible adverse effects of biological therapy with TNF- $\alpha$ antagonists.
\end{abstract}

Key words: skin mucinosis, TNF- $\alpha$ antagonists, adverse effects, biological therapy, psoriasis.

\section{Introduction}

Skin mucinoses are a large group of rare skin diseases in dermatology with undefined pathogenesis and etiology. There are two subgroups of skin mucinosis: those associated with thyroid diseases and those not associated with thyroid disorders. The first group includes skin changes such as generalized and limited myxedema in the course of hypothyroidism, and myxedema accompanying Graves-Basedow disease. A group of mucinoses not associated with thyroid disorders includes scleromyxedema, lichen myxedematosus, sclerosis edema and focal mucinosis [1].

Skin mucinosis is characterized by the distribution of mucin deposits in skin layers. Mucin is a glycosylated protein of high molecular weight. The accumulation of mucin in skin forms glairy material deposits. The histological study with alcian blue and Periodic Acid Schiff (PAS) staining presented mucin deposits mostly in the upper and middle dermis layers, causing the separation of the collagen fibers [1]. The biochemical study shows that the main component of mucin is hyaluronic acid [2-5]. Recent introducing of biological agents into dermatological therapy might be associated with different side effects including skin disorders.

\section{Case report}

A 66-year-old female patient with a 35-year history of psoriasis was admitted to the Department of Dermatology and Venereology, Medical University of Lodz, to be diagnosed and treated for well-demarcated, yellow-brown lesions located on the back (Figure 1). The patient was diagnosed with hypothyroidism in 2006, now in euthyreosis with L-thyroxine at a dose of $100 \mu \mathrm{g}$. For 1 year, the patient was on antibiotic therapy due to inflammation of the urinary tract. The psoriatic skin lesions were mainly located on the patient's lumbar region. The standard systemic therapy which consisted of photochemotherapy, methotrexate and cyclosporine did not give a satisfactory clinical response. The patient was qualified for biological treatment and, thus, underwent therapy with infliximab in standard doses in the period from June 2008 to May 2009. After an initial positive clinical improvement, efficacy of the drug was lost at the $36^{\text {th }}$ week of treatment. The following step was adalimumab therapy (June 2009 - January 2010). Treatment failure forced a change of the biological agent to ustekinumab, which the patient is still receiving. The therapeutic initial response was very good, but there was a subsequent increase in PASI and methotrexate was additionally administered with a satisfac-

Address for correspondence: Assoc. Prof. Aleksandra Lesiak MD, PhD, Department of Dermatology, Medical University of Lodz, 5 Krzemieniecka St, 94-017 Lodz, Poland, phone/fax: +48 4268679 81/+48 42688 45 65, e-mail: aleksandra.lesiak@umed.lodz.pl Received: 7.08.2013, accepted: 1.11.2013. 

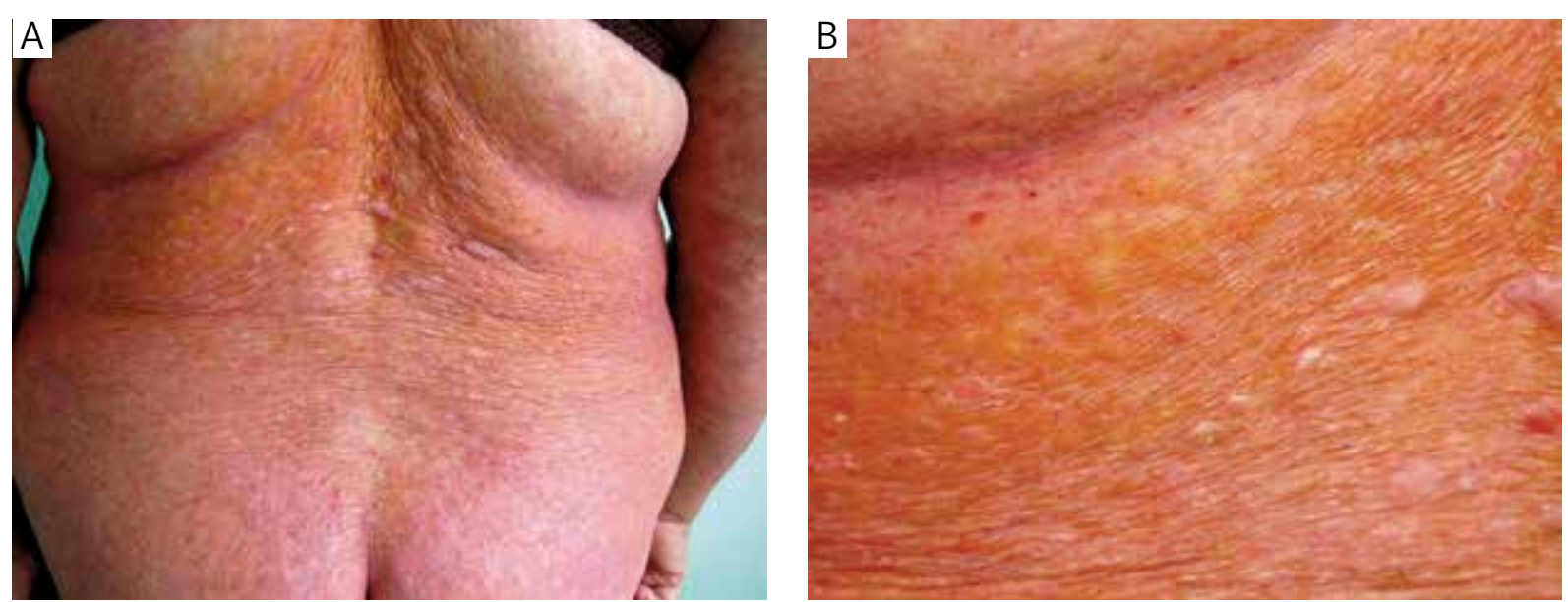

Figure 1. Back localization of papulo-nodular lesions of mucin deposits (A) and well-demarcated, yellow and brown papules and nodules (B)
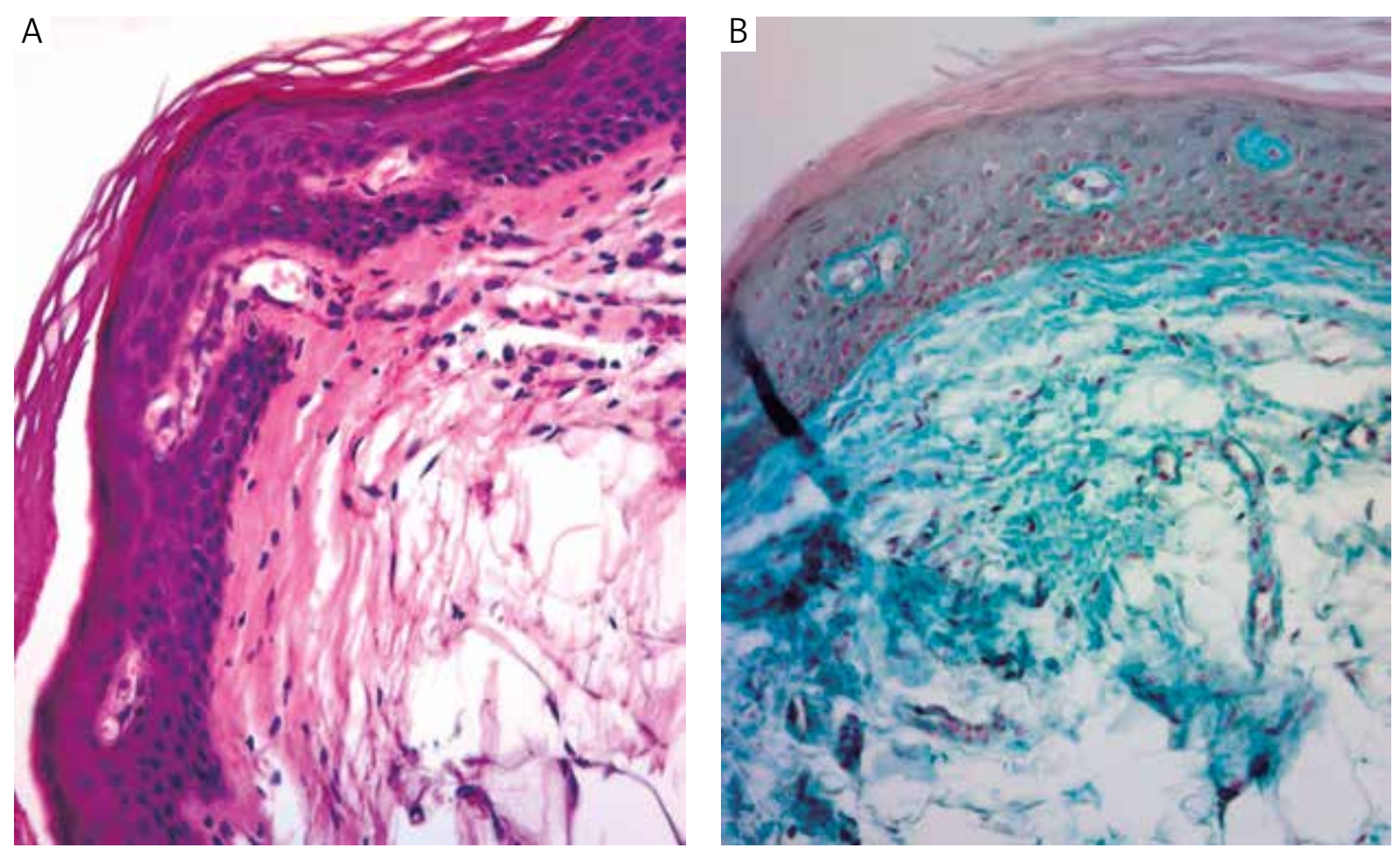

Figure 2. Histopathological examination with (A) hematoxylin-eosin $(H+E$, mag. 100x) and mucin deposits in the dermis in alcian blue (B) (mag. 100x)

tory effect. After 2 months, well-demarcated, yellow and brown, papulo-nodular lesions of 5-10 $\mathrm{mm}$ in diameter were observed on the back. Histopathological examination showed no epidermal changes and staining with alcian blue revealed mucin deposits in the dermis (Figures $2 \mathrm{~A}, \mathrm{~B})$. The laboratory results showed no abnormalities in standard blood morphology. On the basis of clinical and histopathological findings, the diagnosis of cutaneous focal mucinosis was established. The treatment of skin lesions consisted of corticosteroid ointments, which gave little clinical improvement within 2 months. No other adverse effects resulting from the biological therapy were found.

\section{Discussion}

Skin mucinoses are a heterogeneous group of diseases characterized by an accumulation of glycosylated protein in the skin layers. The presented case describes extremely rare coexistence of generalized psoriasis, hy- 
pothyroidism, and focal skin mucinosis. It should be emphasized that a biological therapy could have an impact on the formation of mucin deposits [5].

Skin mucinoses not associated with thyroid diseases are categorized into a separate group. They affect both sexes, but women aged 11-40 years have a higher prevalence [6-8]. The pathogenesis and etiology of the group of non-associated skin mucinosis are currently undefined. There are several hypotheses regarding the factors influencing the induction of mucin deposits in dermis including viral infections, immune disorders and exposure to sunlight [9]. Literature data showed the relationships between skin mucinosis and diseases such as lupus erythematosus, hyperthyroidism, Hashimoto's thyroiditis, diabetes mellitus type 1 , neoplastic hyperplasia, idiopathic thrombocytopenic purpura, myopathy and neuropathy and HIV infection [2, 9-13].

There is a theory which suggests that within skin mucinosis not associated with thyroid disorders an increased synthesis of hyaluronic acid takes place. In one study it was demonstrated that increased levels of hyaluronic acid may be associated with a specific cell line predominantly present in the lesional skin. The level of hyaluronic acid in skin lesions was 2.9-fold higher than in the patient's uninvolved skin, however its synthetic activity in fibroblasts explanted from lesional skin remained unchanged. An immunohistochemical study with the use of anti-factor XIIIa (anti-FXIIIa) antibody demonstrated that the number of FXIIIa+ cells in the lesional skin was significantly increased compared with those in the patient's uninvolved skin and in normal control skin samples [14]. Hyaluronan is thought to be produced by hyaluronic acid synthase (HAS). The cells responsible for the production of hyaluronan acid were also immunohistochemically examined using antibodies against three isoforms of the HAS - HAS1, HAS2 and HAS3. It was revealed that the number of HAS2+ cells was significantly increased in the affected skin $(p<0.01)$. The accumulation of hyaluronan in skin mucinosis may therefore be related to occurrence of elevated populations of dermal plasmacytoid dendritic cells (PDC) with factor XIIIa+/ HAS2+ rather than to dermal fibroblasts [14].

Another study reported that the fibroblasts of patients with skin mucinosis manifest an abnormal response to stimulation by exogenous interleukin IL-1 $\beta$ [6]. The study indicates a role of IL-1 $\beta$ in increased abnormal synthesis of hyaluronic acid in a group of skin mucinosis not associated with thyroid disorders. The detection of inclusions within endothelial cells and pericytes which are typical for virally-affected cells partially provide hypothesis of the role of viral infections in development of skin mucinosis; however, the authors highlight that these bodies can also be produced by high levels of interferon gamma [14].

Mucin synthesis is under control by various cytokines, including transforming growth factor- $\beta$ (TGF- $\beta$ ), interleuk- ins, tumor necrosis factors and interferon $\gamma$. In lung cancer, colon cancer and Hashimoto's thyroiditis, the activated macrophages secrete cytokines in significantly higher amounts than in healthy subjects, which may result in skin fibroblasts stimulation and development of skin lesions characteristic to skin mucinosis [14-16]. The presented case describes cutaneous focal mucinosis development in the course of the psoriatic skin lesions. The exact mechanism leading to mucin deposits formation in psoriatic patients is unclear. Probably production of proinflammatory factors such as IL-1, TNF- $\alpha$, IL-6, IL-8 can activate fibroblasts producing IL-12 and IL-18, and can cause an excessive activation of the synthesis of hyaluronic acid and the creation of mucin deposits in dermis. This phenomenon may occur due to one of the following mechanisms: an abnormal activation of dendritic skin cells XIIIa+/HAS2+ or inadequate response of fibroblasts to stimulation by IL-1 $\beta[16,17]$.

In our case, the possibility of mucinosis induction by anti-TNF- $\alpha$ treatment should be considered. Treatment with TNF- $\alpha$ antagonists is conducted in a relatively short period of time, and the role of anti-TNF- $\alpha$ is not completely defined. One of the hypotheses considers a relation between anti-TNF- $\alpha$, IFN- $\gamma$ and skin dendritic cells. Skin dendritic cells in the absence of active TNF- $\alpha$ produce elevated amounts of IFN- $\alpha$ [18]. The higher level of IFN- $\alpha$ presumably by activation of lymphocytes leads to excessive activation of mucin synthesis by fibroblasts in the skin [17]. Another pathological mechanism which may provoke excessive mucin synthesis is the lack of the inhibitory effect of TNF- $\alpha$ on the population of skin dendritic cells [18].

\section{Conclusions}

Despite ustekinumab treatment in our patient we assume that mucin deposits in this case were provoked by almost 2 years' treatment with TNF- $\alpha$ antagonists. This hypothesis is based on literature data and an extremely short course of ustekinumab treatment. To our knowledge, it is the first case of cutaneous focal mucinosis development in the course of anti-TNF- $\alpha$ therapy. Biologics are more commonly used in dermatological practice, thus new possible adverse effects of biological therapy may be noted.

\section{Acknowledgments}

The study was funded by an internal grant from the Medical University of Lodz no. 503/1-152-01/503-01.

\section{References}

1. Triffer Trevino M, Ahmed I. Plaque-like cutaneous mucinosis (reticular erythematous mucinosis): a clinicopathologic analysis. J Cutan Pathol 1996; 23: 63. 
2. Quimby SR, Perry HO. Plaquelike cutaneous mucinosis: its relationship to reticular erythematous mucinosis. J Am Acad Dermatol 1982; 6: 856-61.

3. Bleehen SS, Slater DN, Mahood J, Church RE. Reticular erythematous mucinosis: light and electron microscopy, immunofluorescence and histochemical findings. Br J Dermatol 1982; 106: 9-18.

4. Greve B, Raulin C. Treating REM syndrome with the pulsed dye laser. Lasers Surg Med 2001; 29: 248-51.

5. Daumerie C, Ludgate M, Costagliola S, Many MC. Evidence for thyrotropin receptor immunoreactivity in pretibial connective tissue from patients with thyroid-associated dermopathy. Eur J Endocrinol 2002; 146: 35-8.

6. Cohen PR, Rabinowitz AD, Ruszkowski AM, DeLeo VA. Reticular erythematous mucinosis syndrome: review of the world literature and report of the syndrome in a prepubertal child. Pediatr Dermatol 1990; 7: 1-10.

7. Adamski H, Le Gall F, Chevrant-Breton J. Positive photobiological investigation in reticular erythematous mucinosis syndrome. Photodermatol Photoimmunol Photomed 2004; 20: 235-8.

8. Rubegni P, Sbano P, Risulo M, et al. A case of reticular erythematous mucinosis treated with topical tacrolimus. $\mathrm{Br}$ J Dermatol 2004; 150: 173-4.

9. Leon-Mateos A, Ginarte M, Leon L, Toribio J. Reticular erythematous mucinosis (REM) with telangiectasisa associated with essential thrombocytosis and lung carcinoma. Eur J Dermatol 2005; 15: 179-81.

10. Steigkeder GK, Kanzow G. Mucin accumulation in the dermis and REM syndrome. Hautarzt Eur I Dermatol 1980; 31: 575-83.

11. Del Pozo J, Pena C, Almagro M, et al. Systemic lapus erythematosus presenting with a reticular erythematous mucinosis-like conditio. Lupus 2000; 9: 144-6.

12. Micalizzi C, Parodi A, Rebora A. Myopathy, destructive arthropathy and peripheral neuropathy in a patient with reticular erythematous mucinosis and monoclonal gammopathy. Dermatology 1999; 199: 371-2.

13. Daudén E, Peńas PF, Buezo GF, et al. Reticular erythematous mucinosis associated with human immunodeficiency virus infection. Dermatology 1995; 191: 157-60.

14. Thareja S, Paghdal K, Lien M, Fenske N. Reticular erythematous mucinosis - a review. Int J Dermatol 2012; 51: 903-9.

15. Izumi T, Tajima S, Harada R, Nishikawa T. Reticular erythematous mucinosis syndrome: glycosaminoglycan synthesis by fibroblasts and abnormal response to interleukin-1 beta. Dermatology 1996; 192: 41-5.

16. Palucka AK, Blanck JP, Bennett L, et al. Cross-regulation of TNF and INF-alpha in autoimmune diseases. Proc Natl Acad Sci USA 2005; 102: 3372-7.

17. Nestle FO, Conrad C, Tun-Kyi A, et al. Plasmacytoid predendritic cells initiate psoriasis through interferon-alpha production. J Exp Med 2005; 202: 135-43.

18. Aeberli D, Oertle S, Mauron H, et al. Inhibition of the TNF-pathway: use of infliximab and etanercept as remission-inducing agents in cases of therapy-resistant chronic inflammatory disorders. Swiss Med Wkly 2002; 132: 414-22. 